\title{
MULTIPLIERS OF FOURIER TRANSFORM \\ IN A HALF-SPACE ${ }^{1}$
}

\author{
BY E. SHAMIR
}

Communicated by F. Browder, September 24, 1964

1. Let $(x, y)$ denote points in $R^{n}$ where $x=\left(x_{1}, \cdots, x_{n-1}\right), y=x_{n}$. Points of the dual space are denoted by $(\xi, \eta)$. Let $Y_{+}$be the characteristic function of the half space $R_{+}^{n}=\{(x, y) \mid y \geqq 0\}$. Let $M(\xi, \eta)$ be an $m \times m$ matrix-valued function whose entries are homogeneous functions:

$$
M_{i j}(\lambda \xi, \lambda \eta)=M_{i j}(\xi, \eta), \quad \lambda>0,1 \leqq i, j \leqq m .
$$

Assume further that $M(\xi, \eta)$ is continuous and nonsingular for $(\xi, \eta) \neq 0$. Consider the bounded operator $M$ in the space $\left(L^{2}\left(R_{+}^{n}\right)\right)^{m}$ (with the natural norm denoted by \|\| ):

$$
M u=Y_{+} \mathcal{F}^{-1}[M(\xi, \eta)(F u)(\xi, \eta)], \quad u \in\left(L^{2}\left(R_{+}^{n}\right)\right)^{m},
$$

where $\mathscr{F}\left(F^{-1}\right)$ denotes the direct (inverse) Fourier transform with respect to all variables. $\mathfrak{F}_{y}\left(\mathfrak{F}_{x}\right)$ will denote the transform with respect to $y$ or $x$ alone. The one-dimensional operator $M_{\xi}$ is similarly defined in $\left(L^{2}\left(R_{+}^{1}\right)\right)^{m}$ with the multiplier $M(\xi, \eta), \xi$ fixed:

$$
M_{\xi} v=Y_{+} \mathcal{F}_{\nu}^{-1}\left[M(\xi, \eta)\left(\mathcal{F}_{y} v\right)(\eta)\right] .
$$

Our main results in this note are the following lemma and theorem.

Lemma. The estimate

$$
\|u\| \leqq C\|M u\|, \quad u \in\left(L^{2}\left(R_{+}^{n}\right)\right)^{m}
$$

holds if and only if for all $|\xi|=1$ (uniformly)

$$
\|v\| \leqq C\left\|\boldsymbol{M}_{\xi}\right\|, \quad v \in\left(L^{2}\left(R_{+}^{1}\right)\right)^{m} .
$$

For the scalar case $(m=1)$, we have

THEOREM. Let $M(\xi, \eta)$ be a homogeneous function continuous and nonvanishing for $(\xi, \eta) \neq 0$. Let

$$
-\frac{1}{2 \pi} \int_{-\infty}^{\infty} d_{\eta} \arg M(\xi, \eta)=k+\theta, \quad k \text { integer, }-1 / 2<\theta \leqq 1 / 2 .
$$

1 The work reported here was supported by the Air Force Office of Scientific Research under Grant No. AFOSR 553-64. 
If $\theta \neq 1 / 2$, then $M$ has $a$ closed range and is injective if $k \geqq 0$, surjective if $k \leqq 0$.

REMARKS. (1) The a priori $L^{2}$-estimates for mixed elliptic problems can be reduced to the validity of (3) [2], [3].

(2) For $n=1, M=M(\eta)$ is determined by $M(1)$ and $M(-1)$. The operator $M$ is then a singular integral operator (with Cauchy kernel) on a half-line. For this case it was shown ([4], cf. also [1], [2], [5]) that $M$ is invertible if and only if the matrix $M(1)^{-1} M(-1)$ does not have real negative eigenvalues.

2. Proof of the Lemma. Assume first that (4) holds, and apply it to

$$
M\left(\frac{\xi}{|\xi|}, \eta\right) \text { and } v(y)=\left(F_{x} u\right)\left(\xi, \frac{y}{|\xi|}\right)
$$

We get

$$
\begin{aligned}
\int_{0}^{\infty} \mid & \left.\left(F_{x} u\right)\left(\xi, \frac{y}{|\xi|}\right)\right|^{2} d y \\
& \leqq C^{2} \int_{0}^{\infty}\left|F_{y}^{-1} M\left(\frac{\xi}{|\xi|}, \eta\right) F_{y}\left[F_{x} u\left(\xi, \frac{y}{|\xi|}\right)\right]\right|^{2} d y \\
& =C^{2} \int_{0}^{\infty}\left|F_{y}^{-1}[M(\xi,|\xi| \eta)|\xi|(F u)(\xi,|\xi| \eta)]\right|^{2} d y \\
& =C^{2} \int_{0}^{\infty}\left|\left(F_{y}^{-1} M F_{u} u\right)\left(\xi, \frac{y}{|\xi|}\right)\right|^{2} d y .
\end{aligned}
$$

After changing variables on both sides (put $\bar{y}=y /|\xi|$ ), cancelling $|\xi|$ and integrating with respect to $\xi$, we have

$$
\int_{R^{n-1}} \int_{0}^{\infty}\left|\left(\mathcal{F}_{x} u\right)(\xi, y)\right|^{2} d y d \xi \leqq C^{2} \int_{R^{n-1}} \int_{0}^{\infty}\left|\left(\mathcal{F}_{y}^{-1} M \mathcal{F} u\right)(\xi, y)\right|^{2} d y d \xi .
$$

Using Parseval's identity (for $\mathcal{F}_{x}^{-1}$ ) we obtain (3).

Assume now that (4) does not hold for some $\bar{\xi}$. Then for any $\epsilon>0$ there is a $v_{\epsilon}(y) \in\left(L^{2}\left(R_{+}^{1}\right)\right)^{m}$ such that $\left\|v_{\epsilon}(y)\right\|=1$ and $\left\|M_{\bar{\xi}} v_{\epsilon}(y)\right\| \leqq \epsilon / 2$. It is easily seen that $\left\|M_{\xi} v_{\epsilon}\right\| \leqq \epsilon$ if $\left|\xi_{R}-\bar{\xi}_{R}\right|<\delta$ and $\delta=\delta(\epsilon)$ is sufficiently small. Let now $w(\xi)$ be the characteristic function of the unit cube and

$$
u_{a}(x, y)=v_{a}(y)(2 \delta)^{-(n-1) / 2 \xi_{x}^{-1} w}\left(\frac{\xi-\xi}{\delta}\right) .
$$


Then $\left\|u_{\epsilon}\right\|=1$ and $\left\|M u_{\epsilon}\right\| \leqq \epsilon$, contradicting (3).

Proof of The Theorem. Solving $M_{\xi} v=w$ is readily seen to be equivalent (via Fourier transform) to solving the Riemann-Hilbert problem

$$
\Phi^{-}(\eta)=M(\xi, \eta) \Phi^{+}(\eta)+\Psi(\eta)
$$

where $\Phi^{ \pm}$are sought in $\left(H_{ \pm}^{2}\left(R^{1}\right)\right)^{m}$, the space of transforms of $L^{2}$-vector functions supported in $R_{ \pm}^{1}$, and $\Psi(\eta)$ is a given $L^{2}$-function. In the scalar case $(m=1)$ this was done by Widom [5, Theorem 3.2]. It follows from Widom's results that if in (5) $\theta \neq 1 / 2$ and $k \geqq 0$ then $M_{\xi}$ is injective and has a closed range for every $\xi \neq 0$, so that (4) is satisfied. It is clear that (4) is satisfied uniformly on the compact set $|\xi|=1$, and by the Lemma we obtain that $M$ is injective and has a closed range. If $\theta \neq 1 / 2$ and $k \leqq 0$ in (5), a consideration of $M^{*}$, the adjoint of $M$, which corresponds to the multiplier $\bar{M}(\xi, \eta)$, shows that $M$ is surjective.

REMARK. It is easily seen that the expression (5) does not depend on $\xi$. Indeed, the homogeneity of $M$ implies that $\lim _{\eta \rightarrow \pm \infty} M(\xi, \eta)$ $=M(0, \pm 1)$, for any $\xi \neq 0$. This means that $\theta$ in (5) is the same for all $\xi$, and by continuity, the same is true for the integer $k$.

\section{REFERENCES}

1. W. Koppelman and J. V. Pincus, Spectral representations for finite Hilbert transformations, Math. Z. 71 (1959), 399-407.

2. J. Peetre, Mixed problems for higher order elliptic equations in two dimensions, Ann. Scuola Norm. Sup. Pisa (3) 15 (1961), 337-353.

3. E. Shamir, Mixed boundary value problems for elliptic equations in the plane, the $L_{p}$ theory, Ann. Scuola Norm. Sup. Pisa (3) 17 (1963), 117-139.

4. - Reduced Hilbert transforms and singular integral equations, J. Analyse Math. 12 (1964), 277-305.

5. H. Widom, Singular integral equations in $L_{p}$, Trans. Amer. Math. Soc. 97 (1960), 131-159.

University of California, Berkeley 